\title{
Effect of Shock Waves Combined With Cytostatics on the Growths of Tumors in vivo
}

\author{
J. ZEMAN ${ }^{1}$, J. BENES ${ }^{1}$, P. POUCKOVA ${ }^{1}$, M. ZADINOVA ${ }^{1}$, P. LUKES ${ }^{2}$ \\ ${ }^{1}$ Institute of Biophysics and Informatics, First Faculty of Medicine, Charles University, Prague, \\ Czech Republic, ${ }^{2}$ Institute of Plasma Physics of the Czech Academy of Sciences Prague, Czech \\ Republic
}

Received July 16, 2019

Accepted October 22, 2019

\section{Summary}

Based on their field of application, the physical parameters of shock waves differ. Experiments referred to in this article used tandem shock waves generated on the surface of a composite anode. There, individual pores of the anode produce multichannel discharges. The composite anode may have a variety of shapes, which, consequently, influence the arrangement of the entire apparatus and the area of their application. Experiments referred to in this article utilise an anode divided into two parts that generated tandem shock waves. The previously conducted experiments have clearly shown that the effect of a tandem shock wave can be very well localized in the focal area, causing necrosis and apoptosis of the tumor cells, and enhancing the effect of cytostatics. This study investigated the effect of tandem shock waves with concomitantly administered cytostatics. We conducted our experiments on Lewis rats. The rats were injected with syngeneic sarcoma tumor cells intradermally and caudally on both the right and left sides. The highest rate of tumor growth inhibition was observed in the cisplatin-treated group that was subsequently treated with shock waves. The effect of shock waves on cell membranes is well described as they increase their permeability due to sonodynamic effect induced by cavitation. The results of experiments referred to in this article conducted in vivo in experimental animals enable us to note that the shock wave increases the effect of chemotherapy administered.

\section{Key words}

Focused shock waves - Double shocks - Cavitation collapse • Tumor damage

\section{Corresponding author}

J. Zeman, Charles University, First Faculty of Medicine, Institute of Biophysics and Informatics, Salmovská 1, Praha 2, 120 00, Czech Republic. E-mail: jan.zeman@If1.cuni.cz

\section{Introduction}

Shock waves have been used in medicine for more than 30 years not only in the treatment of concrements in the form of their fragmentation but also in other fields. It consists of a short pulse that only lasts several $\mu \mathrm{s}$. In the beginning, it almost immediately achieves the peak value of the positive pressure $p+$; this transition takes about $10 \mu$ s and is called the "shock". Depending on the tip, the positive pressure value reaches about 10-150 MPa. Subsequently, the pressure drops to zero, followed by a negative pressure lasting about $3 \mu \mathrm{s}$. The negative pressure value, $\mathrm{p}$-, can reach -25 $\mathrm{MPa}$. Thus, the negative pressure is lower than the positive pressure, and, at the same time, there occurs no "shock" change in this phase. Even though the entire course is generally referred to as the shock wave, from the technical point of view, it is only the sharp transition of pressure at the beginning of the process (Loske 2010, Lukes et al. 2016, Coleman et al. 1993, Gerdesmeyer et al. 2002, Smith 2012).

The earliest application of shock waves in medicine focused on fragmentation of concrements; in this case, concrements fragmentation without damaging the soft tissue. The shock wave must pass through the soft tissue in order to reach the concrement where it can cause damage. Usually, the appearance of several hematomas during the treatment of kidney stones represents the first indication of such damage. Additionally, haematuria was also noticed in this type of 
treatment that was attributed to microscopic lesions, which were caused by stone fragments accelerated by a shock wave. Discrete renal transformations following the shock wave treatment were also confirmed by other experiments conducted in vivo on animals. Shock waves and their propagation and effects in tissue should be predictable, as we can describe them physically. However, as they propagate in biological systems, the matter is much more complicated. Although in the lithotripsy, the shock wave is aimed at the concrement, the adjacent tissue is also exposed to its effects. The length of the focal volume of many lithotriptors is about $50 \mathrm{~mm}$, and this means that the shock waves affect the entire thickness of the kidneys. At the same time, respiratory movements, as well as movements of the patient can cause displacement of the concrement out of the focus area, and the soft tissue is, thus, exposed to the shock waves. Fortunately, the tissue has specific physical properties that make it much less prone to damage caused by shock waves. For example, the acoustic impedance of the tissue and water is very close, and, therefore, the shock wave passes through the water-tissue interface almost without reflection.

Two types of bleeding can be distinguished in the kidneys: diffuse bleeding and hematoma. Kidneys have been one of the most studied organs, and similar changes have been attested in most other tissues. Pulmonary tissue represents one of the exceptions. Pulmonary bleeding was one of the earliest documented undesirable effects of shock wave treatment. It happened when the shock wave targeted the gallbladder. Pulmonary tissue has a different acoustic impedance and, therefore, has been the most sensitive to shock waves in terms of damage. Moreover, mild damages to liver, gallbladder, intestine and muscle tissue, as well as kidney, have also been documented.

Another medically beneficial effect of the shock waves can be attested on bones. As expected, bleeding represented the main effect a shock wave had on the bone. Haemorrhage was observed in the periosteum and also in the bone marrow. The bone itself, however, responded quite unexpectedly to the damage, namely an intense apposition of new bone at the site of application, the cortical layer was considerably thickened.

A substantial effect that had to be examined was the effect on the heart and nerve cells. Already at the beginning of the application of this treatment on people, the occurrence of cardiac arrhythmias has been recorded. It can be prevented by triggering the shock wave outside the vulnerable phase of the cardiac cycle. In practice, it meant monitoring the patient's ECG and triggering the shock waves to synchronise them with the R-wave. In vitro experiments have also shown that shock waves can induce an action potential. When treating the patients, muscle tissue twitching has been therefore observed (Smith 2012, Chaussy et al. 1982, Delius et al. 2002, McClure et al. 2003).

Shock waves create cavitation in the tissues, which has been corroborated by diagnostic ultrasound. They were detected as flashing areas in the high-pressure field. Already in the 1980s, the hypothesis that cavitation was responsible for tissue damage was being evaluated and tested. Several findings have supported it.

A correlation between shock wave repetition and soft tissue damage has been corroborated. During the patient's treatment, 500-3000 shock waves are usually generated, with a frequency of one or two waves per second. In order to shorten the treatment time, the more rapid repetition of the shock waves has been proposed. The treatment time would then be reduced to a few minutes. Such a possibility had been experimentally investigated, and it was corroborated that shortening of intervals between shocks means a more significant degree of tissue damage. It was caused by the fact that the cavitation created by the first shock wave and the subsequent acoustic changes in its surroundings last longer and might react with a second shock wave that came in a very short interval. Similar experiments confirmed the negative effect of shortening the interval, and, therefore, the idea of shortening of the interval between individual shock waves was abandoned.

It was also detected that if microbubbles were administered at the same time as the shock waves, the bleeding increased. Moreover, it was corroborated that the tissue was being damaged in areas where ultrasound detected the cavitation (Wolfrum 2004, McClure et al. 2003, Shima 1997).

Shock waves, as demonstrated, can interact with tissue. In the case of lithotripsy, it is desirable not to damage the surrounding tissue, which would be clinically significant compared to the effect of the treatment. However, there are also areas of shock wave application where the potential increase in the effects on the tissue can be utilised, such as in oncology.

Adverse effects can be increased by cavitation enhanced by elevating the negative pressure in focus; however, such a mechanism has its limitations. Even though the shock waves can be applied in a quicker 
sequence, it seems that the cavitation generated by the first shock wave appears to dampen the second wave negatively. Moreover, experiments have concluded that should the shock sequence be shorter than 10 microseconds, this effect began to take place and is more defined (Sunka et al. 2007, Benes et al. 2007). First of all, it increases the permeability of phospholipid membranes, which is crucial for cell survival. However, for medical applications, it is sometimes necessary to circumvent the control mechanisms so that selected molecules or genes can be delivered to the cell. Currently, there are several systems on how to deliver something to a cell, and the choice of application system depends on the situation. Physical systems include electroporation and optoporation. However, it is also possible to use shock waves; such a method is called sonoporation and is essential for in vivo administration. It has been corroborated that lithotripter may facilitate penetration of the cell membrane to molecules that would not usually be able to penetrate it. Thus, procedures known from lithotripsy (Wolfrum 2004) can be used to administer medicinal substances.

For example, the experiments corroborated that shock waves enhanced the effect of Cisplatin. If the cells are contained in an over-pressured vessel, the permeability of their membranes, under shock waves, is reduced in comparison to the condition at standard pressure. This observation leads to the conclusion that the membrane pores are caused by cavitation. It has been experimentally verified that it is possible to deliver protein-inactivating ribosomes to a tumor cell, and, thus, to reduce the dose of drug administered up to 40,000 times (Kodama et al. 2003). Several megadalton plasmids that encode standard enzymes such as galactosidase or luciferase may also be transferred.

This effect on cell membranes, i.e. the sonoporation, can be utilised in conjunction with chemotherapy. Concerning in vivo therapy, it appears from the experiments performed that the shock wave has an effect on microcirculation in the tumor, and, thus, perfusion is reduced. Possible clinical use of this treatment is currently being tested (Delius 2002, Lauer et al. 1997, Ohl et al. 2006, Kodama et al. 2003, Ohl et al. 2003).

This study focuses on the effect of shock waves on tumor tissue in combination with cisplatin in vivo. In our experiment, we studied the effect of shock waves, as well as cisplatin, administered separately and in combination, on tumor tissue growth over time.

\section{Methods}

In the experiment, shock waves have been generated by a new source based on the principle of multichannel discharge. This method of generating shock waves was newly developed in cooperation with the Institute of Plasma Physics of the CAS. In the course of the development of the device, we applied the knowledge gained from the research of impulse corona discharges, which had been initially intended for water purification. The way how the volume corona discharge is generated is subject to a patent protection (Sunka et al. 2002, Sunka et al. 1997). The principle of the shock wave generation is that the space between the two electrodes is filled with liquid with increased electrical conductivity. A multichannel discharge occurs after the impulse voltage is applied to the electrode that has the shape of the required wavefront. Such electrical discharge burns between the electrode and the liquid. Thus, individual quasi-spherical pressure waves are formed, and their superposition creates one powerful pressure wave. It is further focused with a reflector, and on its way to the focus, it is transformed into a shock wave.

The multichannel discharge, as shown in Fig. 1, occurs on the surface of the composite anode. The stainless steel cylinder is coated with a thin layer (0.2-0.5 mm) of porous ceramics with an open porosity of 3-5 \%. The second electrode is a reflector. After connecting the impulse voltage source to the electrode system, the discharges can be concentrated into individual pores. Inside these pores, an electric field with an intensity of $\mathrm{E} \sim 0.5-1 \mathrm{MV} / \mathrm{cm}$ arises, and discharge is generated in the pores at the given intensity level. By using a liquid with increased conductivity $(5-20 \mathrm{mS} / \mathrm{cm})$, the discharges are concentrated in the individual pores of the insulation layer, and the discharge generates a cylindrical pressure wave.

With this generator, shock waves are gradually generated from the pressure wave by passing through the environment. By setting the circuit parameters such as voltage, capacitor capacity, circuit inductance, as well as liquid conductivity, the generator enables influencing the properties of the shock waves (amplitude, time course). Because the discharge current is divided into many pores, erosion of the composite anode is reduced, and its service life is extended.

Fig. 2 contains a diagram of the generator used for generating a tandem shock wave. 


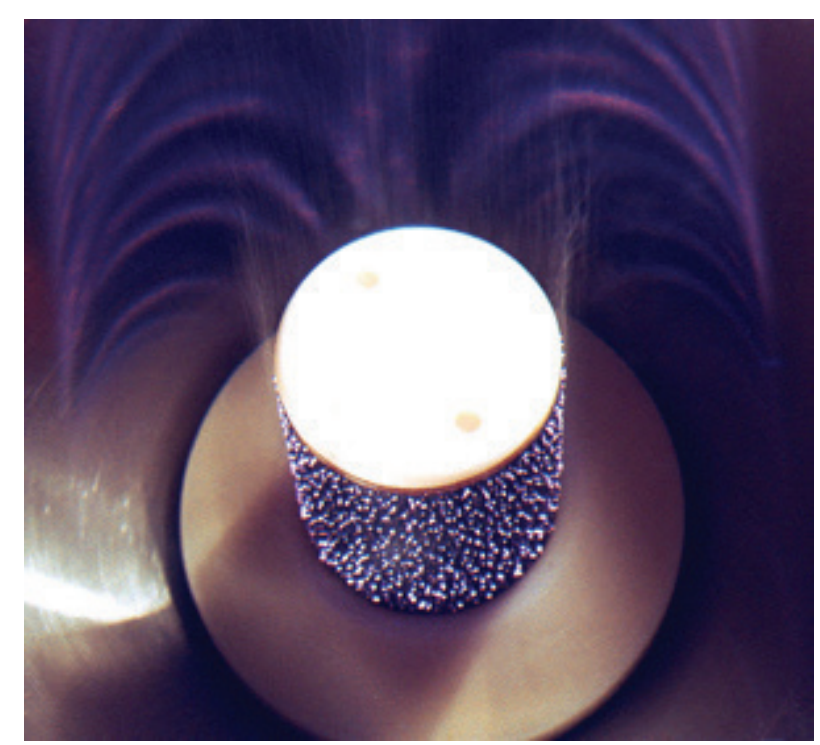

Fig. 1. Composite anode at the time of discharge.

In this case, the composite anode consists of two parts (1: $60 \times 70 \mathrm{~mm}$ in $\varnothing ; 2: 77 \times 25 \mathrm{~mm}$ in $\varnothing)$. Each of the composite anode sections can be switched on separately, and, thus, generate tandem shock waves with different delays between the first and second waves. The time delay can also be determined by the geometry of the individual segments of the electrode. It means that if both segments are switched on simultaneously, the second shock wave is delayed continuously by $5 \mu$ s due to different pressure wave paths to the focus. In this case, the power circuit is more straightforward.

We used Lewis rats in the experiment. Their weight ranged from 200-250 g. They were injected with syngeneic sarcoma tumor cells intradermally and caudally on both the right and left sides. Subsequently, we divided the rats into groups, each comprising 30 animals; thus, a total of 90 animals was used.

1) The first group of rats was exposed to shock waves;

2) The other group received a dosage of $5 \mathrm{mg} / \mathrm{kg}$ of cisplatin prior to application of shock waves. Consecutively, they were subjected to shock waves;

3) The third group received only a dosage of $5 \mathrm{mg} / \mathrm{kg}$ of cisplatin.

A tumor on the other side of the animal was always used as a control. Altogether, 120 shocks were applied at an angle of $60^{\circ}$ and 120 shocks at an angle of $120^{\circ}$ with respect to the axis of the animal in one plane. The shock waves were applied so that the focus did not interfere with the control tumor or another organ. Subsequently, the volume of tumor tissue was measured

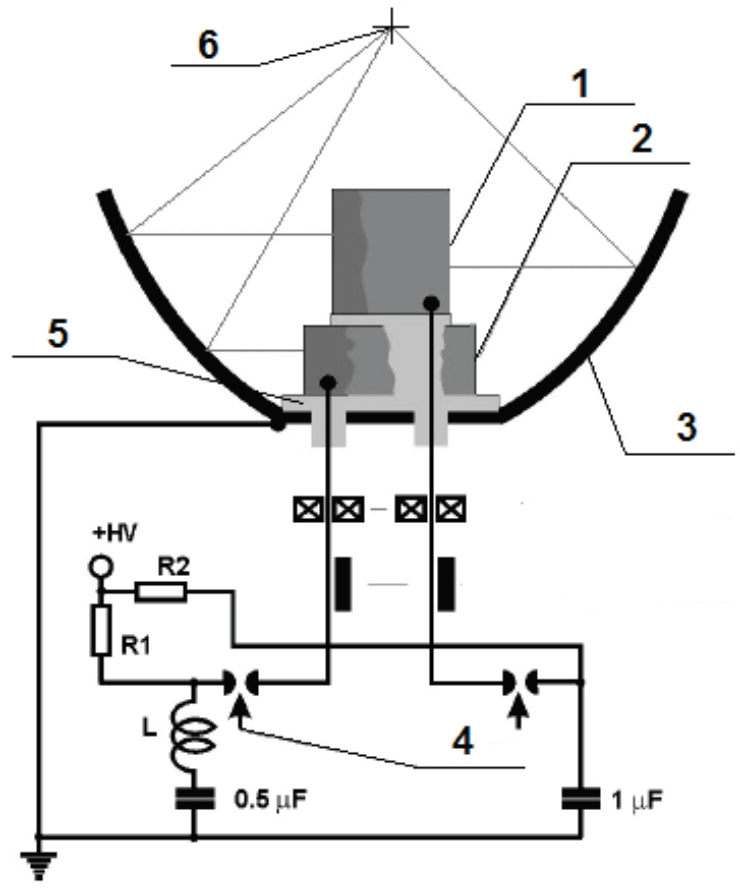

Fig. 2. Schematic diagram of a shock wave generator with a tandem composite anode. 1: Composite anode with a smaller diameter; 2: CFomposite anode with a larger diameter; 3: Reflector; 4: Spark-gap; 5: Insulator; 6: Focus.

over time. The volume $\mathrm{V}$ was calculated as $\mathrm{V}=\mathrm{a} \times \mathrm{b}^{2} \times \pi / 6$, where $a$ is the length of the tumor, and $b$ its width. The dimension measurements were made by using a digital calliper.

\section{Results}

Our experiment focused on the effect of tandem shock waves on syngeneic sarcoma growth in vivo applied in combination with cisplatin, as well as separately. Lewis rats were injected with syngeneic sarcoma tumor cells intradermally to their right and left flank, followed by tandem shock waves combined with cisplatin application, as well as separately; followed by measurements of tumor volumes $\mathrm{V}$ on $1^{\text {st }}, 3^{\text {rd }}, 5^{\text {th }}, 7^{\text {th }}, 9^{\text {th }}$, and $11^{\text {th }}$ day after the application of tandem shock waves. Mean values and standard deviations of these values were recorded. Chart 1 illustrates how the tumor volume varied with respect to the default values for each group.

After cisplatin administration (Chart 1), the tumor growth slowed down compared to the control group. However, growth was faster compared to the group that was only treated with shock waves. The highest level of tumor growth inhibition was observed in the cisplatin-treated group that was subsequently treated with tandem shock waves. There, the tandem shock wave worked 


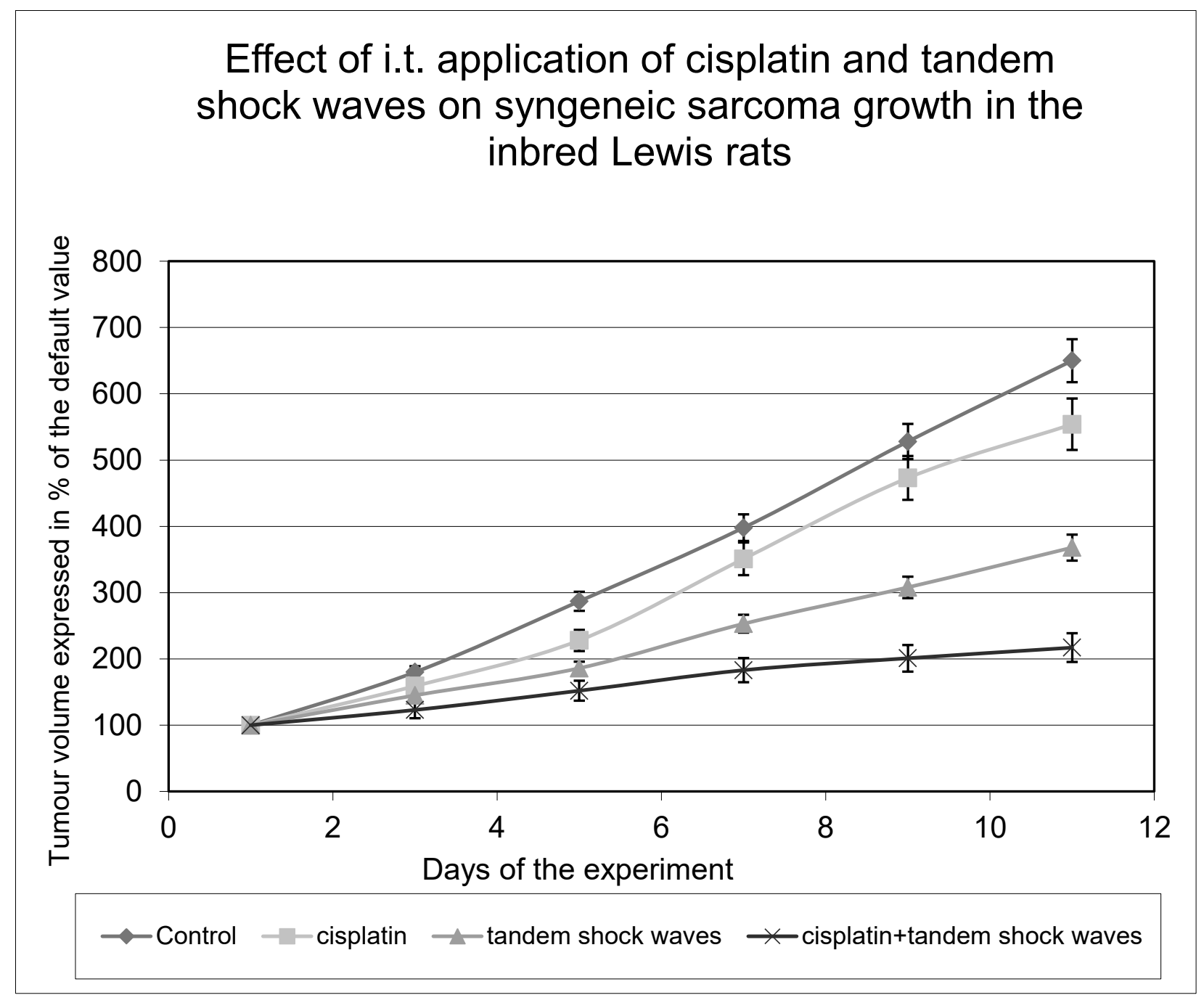

Chart. 1. Tumor volumes measured in individual days

together with cisplatin and most significantly slowed the tumor growth.

\section{Discussion}

Nowadays, the shock wave treatment is commonly used in rheumatology, and we may say that the number of indications exceeds the application in the field of urology. Shock waves are also used in orthopaedics for the treatment of poorly healing fractures. Moreover, it has been experimentally used to treat ischemic heart disease, as well as erectile dysfunctions. Last but not least, shock waves can also be used to deliver different molecules into the cell and penetrate the cellular membranes.

There is an interest in shock waves also in the field of oncology, where it would be plural to cause damage to tumor tissue, and, therefore, to help the effect of chemotherapeutic agents.
This study focused on the possibilities of using tandem shock waves in oncology. Shock waves are generated by a new type of generator based on the principle of multichannel discharge on the surface of a composite anode. The composite anode can be divided into two segments, and, thus, to generate a tandem wave.

The experiments have clearly shown that tandem shock waves are capable of damaging the tumor tissue and, thus, leading to a slowdown in tumor growth. However, its ability to increase the effect of cytostatics, the cisplatin in the case of our experiment, is more important. The application of tandem shock waves is still in its experimental phase, but so far it is the only energy that can be concentrated to a small target area without damaging the surrounding tissues, and it is irrelevant whether the effect is caused only by the direct toxic effect of the tandem shock waves, or the molecules would be activated by cavitation or micelles disrupted by the cytostatics. 
These effects of shock waves have also been confirmed in studies conducted by other authors (Lauer et al. 1997, Ohl et al. 2006, Kodama et al. 2003, Ohl et al. 2003). The effect of shock waves on cell membranes has also been well described. Shock waves increase cell membrane permeability due to sonodynamic effect induced by cavitation. Such an effect, therefore, promotes the penetration of cisplatin into the intracellular environment. Mechanical stress can also play a role in increasing cell membrane permeability, as shown by experiments performed with ultrasound. (Bernard et al. 2012). Thus, the shock waves appear to be a promising tool for promoting chemotherapy and also for introducing other macromolecular substances into the intracellular environment (Lopez-Marin et al. 2018).

\section{Conflict of Interest}

There is no conflict of interest.

\section{Acknowledgements}

This work was supported by the Ministry of Education, Youth and Sports of the Czech Republic (CZ.1.05/3.1.00/14.0299).

\section{References}

BENES J, SUNKA P, KRALOVA J, KASPAR J, POUCKOVA P: Biological effects of two successive shock waves focused on liver tissues and melanoma cells. Physiol Res 56 (Suppl 1): S1-S4, 2007.

BERNARD V, MORNSTEIN V, SKORPIKOVA J, JAROS J: Ultrasound and cisplatin combined treatment of human melanoma cells A375 - the study of sonodynamic therapy. Ultrasound Med Biol 38: 1205-1211, 2012.

COLEMAN AJ, SAUNDERS JE: A review of the physical properties and biological effects of the high amplitude acoustic fields used in extracorporeal lithotripsy. Ultrasonics 31: 75-89, 1993.

DELIUS M: Twenty years of shock wave research at the institute for surgical research. European Surg Res 34: 30-36, 2002.

GERDESMEYER L, MAIER M, HAAKE M, SCHMITZ C: Physikalisch-technische Grundlagen der extrakorporalen Stoßwellentherapie (ESWT). Der Orthopäde 31: 610-617, 2002.

CHAUSSY C, SCHMIEDT E, JOCHAM D, BRENDEL W, FORSSMANN B, WALTHER V: First clinical experience with extracorporeally induced destruction of kidney stones by shock waves. J Urol 197: 2S, 2017.

KODAMA T, DOUKAS AG, HAMBLIN MR: Delivery of ribosome-inactivating protein toxin into cancer cells with shock waves. Cancer Lett 189: 69-75, 2003.

LAUER, U, BÜRGELT E, SQUIRE, Z MESSMER K, HOFSCHNEIDER PH, GREGOR M, DELIUS M: Shock wave permeabilization as a new gene transfer method. Gene Therapy 4: 710-715, 1997.

LÓPEZ-MARÍN LM, RIVERA AL, FERNÁNDEZ F, LOSKE AM: Shock wave-induced permeabilization of mammalian cells. Phys Life Rev 26-27: 1-38, 2018.

LOSKE AM: The role of energy density and acoustic cavitation in shock wave lithotripsy. Ultrasonics 50: 300-305, 2010.

LUKES P, SUNKA P, HOFFER P, STELMASHUK V, POUCKOVA P, ZADINOVA M, ZEMAN J, DIBDIAK L, KOLAROVA H, TOMANKOVA K, BINDER S, BENES J: Focused tandem shock waves in water and their potential application in cancer treatment. Shock Waves 24: 51-57, 2014.

LUKES P, FERNÁNDEZ F, GUTIÉRREZ-ACEVES J, FERNÁNDEZ E, ALVAREZ UM, SUNKA P, LOSKE AM: Tandem shock waves in medicine and biology: a review of potential applications and successes. Shock Waves 26: 1-23, 2016.

MCCLURE S, DORFMÜLLER C: Extracorporeal shock wave therapy: Theory and equipment. Clin Tech Equine Practice 2: 348-357, 2003.

OHL C-D, WOLFRUM B: Detachment and sonoporation of adherent HeLa-cells by shock wave-induced cavitation. Bioch Biophys Acta (BBA) 1624: 131-138, 2003.

OHL C-D, ARORA M, IKINK R, DE JONG N, VERSLUIS M, DELIUS M, LOHSE D: Sonoporation from Jetting Cavitation Bubbles. Biophys J 91: 4285-4295, 2006.

SHIMA A: Studies on bubble dynamics. Shock Waves 7: 33-42, 1997. 
SMITH AD, BADLANI GH, PREMINGER GM, KAVOUSSI LR (eds): Smith's Textbook of Endourology, Oxford, UK, Wiley-Blackwell, 2012.

SUNKA P, STELMASHUK V, BENES J, POUCKOVA P, KRALOVA J: Potential applications of tandem shock waves in cancers therapy. In: 2007 16th IEEE International Pulsed Power Conference. IEEE, 1074-1077, 2007.

SUNKA P, BABICKY V, CERMAK M, CLUPEK M: Method of generating espacious corona discharge in water and apparatus for making the same. CZ9601596. CZ. Patent Document. 1997-08-13.

SUNKA P, BABICKY V, CLUPEK M, NEUFUSS K, BENES J: Generation method of focused shock wave by electric discharge in a liquid and apparatus for making the same. CZ20001513. CZ. Patent Document. 2002-08-14.

WOLFRUM B: Cavitation and shock wave effects on biological systems. Gottingen, 2004. Dissertation. GeorgAugust-Universitat zu Gottingen. 